\title{
Measurement of $\sigma(t \bar{t} b \bar{b}) / \sigma(t \bar{t} j j)$ ratio at $13 \mathrm{TeV}$ with the CMS Detector
}

\section{Young-kwon Jo*t}

on behalf of the CMS Collaboration

E-mail: young-kwon.jo@cern.ch

The measurement of the cross section ratio $\sigma(t \bar{t} b \bar{b}) / \sigma(t \bar{t} j j)$ is presented using a data sample corresponding to an integrated luminosity of $2.3 \mathrm{fb}^{-1}$ collected in pp collisions at $\sqrt{s}=13 \mathrm{TeV}$ with the CMS detector at the LHC. Events with two leptons and at least four reconstructed jets, including at least two identified as b quark jets, in the final state are selected. The measured ratio is $0.022 \pm 0.003$ (stat.) \pm 0.006 (syst.) in the full phase space. The measured cross section $\sigma(t \bar{t} b \bar{b})$ is $3.9 \pm 0.6$ (stat.) \pm 1.3 (syst.) pb and $\sigma(t \bar{t} j j)$ is $176 \pm 5$ (stat.) \pm 33 (syst.) pb.

38th International Conference on High Energy Physics 3-10 August 2016

Chicago, USA

* Speaker.

${ }^{\dagger}$ Korea University 


\section{Introduction}

After the Higgs boson discovery [1,2], the measurement of its couplings to the other standard model (SM) particles, in particular to the top quark, is crucial component of the physics program at the LHC. One of the most promising channels for a direct measurement of the top quark Yukawa coupling in the SM is the production of the Higgs boson in association with a $t \bar{t}$ pair $(t \bar{t} H)$, where the Higgs boson decays to $b \bar{b}$, thus leading to a $t \bar{t} b \bar{b}$ final state as like Figure 1.
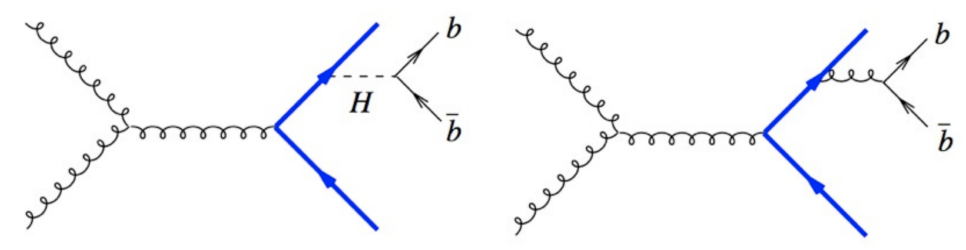

Figure 1: The diagrams are typical diagrams for $t \bar{t} H$ (left) and $t \bar{t} b \bar{b}$ (right).

It is crucial to understand the $t \bar{t} b \bar{b}$ processes and $t \bar{t} j j$ process. $t \bar{t} b \bar{b}$ is the main irreducible background for $t \bar{t} H$ searches. $t \bar{t} j j$ is a reducible background as contributes through the misidentification of $b$-jets. The primary motivation for measuring the cross section ratio is that there are common systematic uncertainties for $t \bar{t} b \bar{b}$ and $t \bar{t} j j$ that cancel out such as the uncertainty on the integrated luminosity. The measurements of the cross sections $\sigma(t \bar{t} b \bar{b}), \sigma(t \bar{t} j j)$, and their ratio are presented using the dilepton final states with a data sample of pp collisions collected in 2015 with a centre-of-mass energy of $13 \mathrm{TeV}$ by the CMS experiment [5].

\section{Signal definition}

At generator level we define the physics objects as:

- Lepton $(\mu, e)$ : particles from the decay of $\mathrm{W}$ bosons with $p_{T}>20 \mathrm{GeV}$, and $|\eta|<2.4$

- Gen-jet of anti- $k_{T}$ algorithm with $\mathrm{R}=0.4$ built with the $p_{T}>20 \mathrm{GeV}$, and $|\eta|<2.5$.

- The jets are clustered using all stable particles (except neutrinos) and are checked to have $\Delta R>0.5$ with respect to the selected leptons.

In the visible phase space, the $t \bar{t}$ events are categorized as:

$$
\begin{array}{ll}
t \bar{t} b \bar{b}: \text { two leptons }+4 \text { b-jets } & t \bar{t} b j: \text { two leptons }+3 \text { b-jets }+1 \text { jet } \\
t \bar{t} c \bar{c}: \text { two leptons }+2 \text { b-jets }+2 \text { c-jets } & t \bar{t} L F: \text { two leptons }+2 \text { b-jets }+2 \text { jets } \\
t \bar{t} j j: t \bar{t} b \bar{b}+t \bar{t} b j+t \bar{t} c \bar{c}+t \bar{t} L F &
\end{array}
$$

\section{Data and MC samples}

Data are selected using dilepton triggers and compared to simulated signal and background MC samples; We use the following simulations: $t \bar{t}($ POWHEG), single top(POWHEG), DYJets(aMC@ NLO), WJets(aMC@NLO), Di-boson(PYTHIA8), and $t \bar{t} \mathrm{~V}(\mathrm{aMC} @ \mathrm{NLO})$ 


\section{Event selection}

Two oppositely charged isolated and well identified leptons (electron or muon) with $p_{T}>20$ $\mathrm{GeV}$ and $|\eta|<2.4,76 \mathrm{GeV} \geq M_{\ell \ell} \geq 106 \mathrm{GeV}$ and missing $E_{T}>40 \mathrm{GeV}$ for $e e, \mu \mu$ channel, with at least 4 jets with $p_{T}>30 \mathrm{GeV}$ and $|\eta|<2.4$ by using anti- $k_{T}(\mathrm{R}=0.4)$ algorithm, and at least 2 b-tagged jets identified with the combined secondary vertex (CSV) algorithm [3].

\section{Measurement}
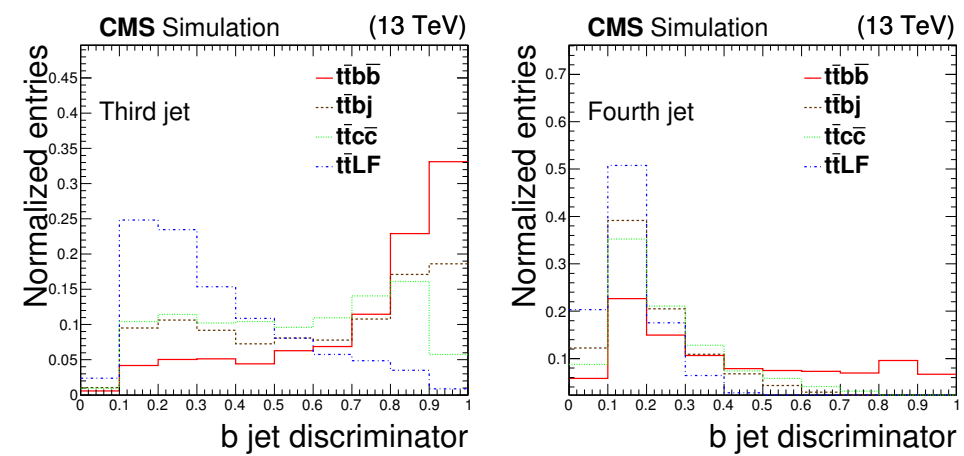

Figure 2: The distributions are normalized shapes of the b-jet discriminator for $3^{\text {rd }}$ and $4^{\text {th }}$ jets for each $t \bar{t} b \bar{b}, t \bar{t} b j, t \bar{c} c \bar{c}$, and $t \bar{t} L F$ contributions.

A binned maximum likelihood fit is performed to the two-dimensional distribution of the btagging discriminators(Fig. 2) of the $3^{\text {rd }}$ and $4^{\text {th }}$ jets simultaneously.

The number of expected events is parameterised as follows:

$f(k, R)=k \cdot N_{t \bar{t} j j} \cdot\left[R \cdot N_{t \bar{t} b \bar{b}}^{n o r m}+R^{\prime} \cdot N_{t \bar{t} b j}^{n o r m}+\left(1-R-R^{\prime}\right) \cdot N_{t \bar{t} L F / t \bar{t} \bar{c} \bar{c}}^{n o r m}\right]+k \cdot N_{t \bar{t} \text { others }}+N_{b k g}^{M C}+N_{b k g}^{\text {Data-driven }}$,

where $\mathrm{R}$ is the $t \bar{t} b \bar{b} / t \bar{t} j j$ ratio, and $R^{\prime}$ is the $t \bar{t} b j / t \bar{t} j j$ ratio at reconstruction level. An overall signal normalization scale factor $(k)$ is allowed to float freely in the fit. Fig. 3 summarises the distributions of the $\mathrm{b}$ jet discriminators in data, after the fit is performed.

\section{Result}

We measured the cross sections as $\sigma(t \bar{t} b \bar{b}), \sigma(t \bar{t} j j)$ and cross section ratio $\sigma(t \bar{t} b \bar{b}) / \sigma(t \bar{t} j j)$ in the visible and the full phase space summarised in Table 1 Table 1. Result are compared with POWHEG+PYTHIA8-based predictions.

\section{Conclusions}

We have measured the $t \bar{t} b \bar{b}$ and $t \bar{t} j j$ cross sections and its ratio at $13 \mathrm{TeV}$ for the first time using 2.3 $\mathrm{fb}^{-1}$ of data taken by the CMS detector in 2015 . The measurements are performed in the visible and full phase space. The $\sigma(t \bar{t} b \bar{b}) / \sigma(t \bar{t} j j)$ ratio is compatible with the expectation obtained from 

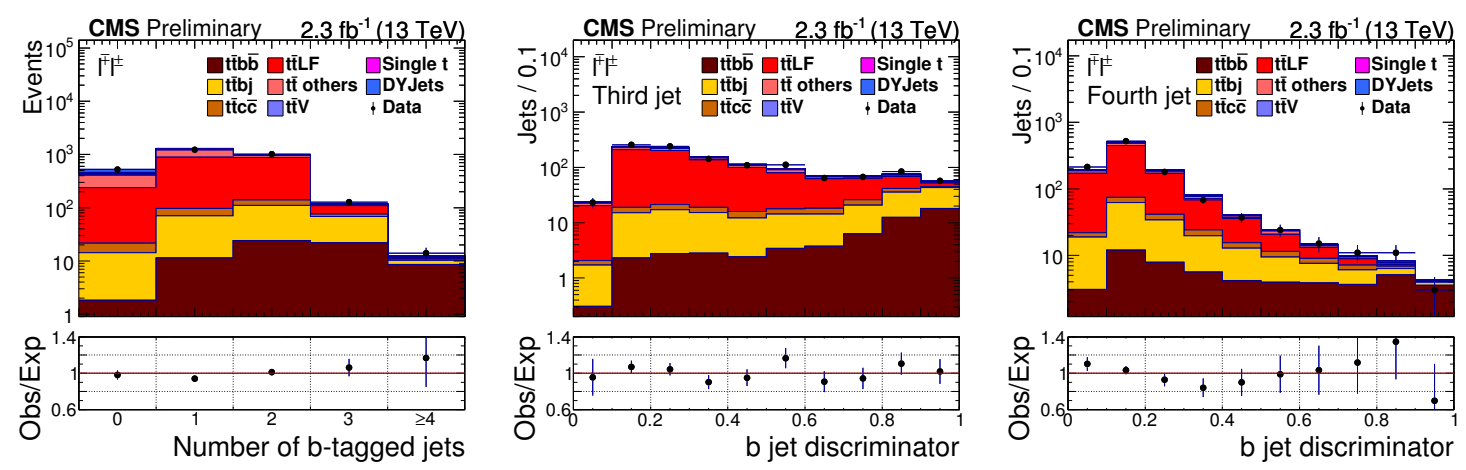

Figure 3: The distributions are shapes of b-jet multiplicity(left) and b-tagging discriminators of the $3^{\text {rd }}$ (center) and $4^{\text {th }}$ (right) jets, after performing the fit described in the text. The separate contributions from the $t \bar{t} b \bar{b}, t \bar{t} b j, t \bar{t} c \bar{c}$, and $t \bar{t} L F$ components are shown separately.

\begin{tabular}{l|ccc}
\hline \hline Phase Space & $\sigma(t \bar{t} b \bar{b})[\mathrm{pb}]$ & $\sigma(t \bar{t} j j)[\mathrm{pb}]$ & $\sigma(t \bar{t} b \bar{b}) / \sigma(t \bar{t} j j)$ \\
\hline Measurement & & & \\
Visible & $0.085 \pm 0.012 \pm 0.029$ & $3.5 \pm 0.1 \pm 0.7$ & $0.024 \pm 0.003 \pm 0.007$ \\
Full & $3.9 \pm 0.6 \pm 1.3$ & $176 \pm 5 \pm 33$ & $0.022 \pm 0.003 \pm 0.006$ \\
\hline Simulation $(P O W H E G)$ & & & \\
Visible & $0.070 \pm 0.009$ (scale) & $5.1 \pm 0.5$ (scale) & $0.014 \pm 0.001$ (scale) \\
Full & $3.2 \pm 0.4$ (scale) & $257 \pm 26$ (scale) & $0.012 \pm 0.001$ (scale) \\
\hline \hline
\end{tabular}

Table 1: The table is shown cross section and cross section ratio with statistics $\left(1^{\text {st }}\right)$ and systematic $\left(2^{\text {nd }}\right)$ uncertainty in measurement and simulation.

the POWHEG simulation. This result helps to understand the main background in the search for $t \bar{t} \mathrm{H}$ and to test the validity of NLO QCD calculations at $\sqrt{s}=13 \mathrm{TeV}$.

More details of this measurement can be found in [4] .

\section{References}

[1] ATLAS Collaboration, Observation of a new particle in the search for the Standard Model Higgs boson with the ATLAS detector at the LHC. Phys. Lett. B, 1:716, 2012.

doi:10.1016/j.physletb.2012.08.020

[2] CMS Collaboration, Observation of a new boson at a mass of $125 \mathrm{GeV}$ with the CMS experiment at the LHC. Phys. Lett. B, 30:716, 2012.

doi:10.1016/j.physletb.2012.08.021

[3] CMS Collaboration, Identification of b-quark jets with the CMS experiment. Journal of Instrumentation, 8:P04013, 2013.

http://stacks.iop.org/1748-0221/8/i=04/a=P04013

[4] CMS Collaboration, Measurement of ttbb production at 13 TeV. CMS-PAS-TOP-16-010, 2016.

https://cds.cern.ch/record/2202803

[5] CMS Collaboration, The CMS experiment at the CERN LHC, JINST 3 (2008) S08004. doi:10.1088/1748-0221/3/08/S08004 